\title{
ROTOR LOSSES IN LAMINATED MAGNETS AND AN ANISOTROPIC CARBON FIBER SLEEVE
}

\author{
M. van der Geest, J.J. Wolmarans, H. Polinder, J.A. Ferreira *, D. Zeilstra ${ }^{\dagger}$ \\ ${ }^{\star}$ Delft University of Technology, Delft, The Netherlands, ${ }^{\dagger}$ Aeronamic BV, Almelo, The Netherlands \\ email:m.vandergeest@tudelft.nl
}

Keywords: Carbon fiber, Magnet Lamination, Rotor Losses, 3D FEM.

\begin{abstract}
High speed fault tolerant permanent magnet machines have strong asynchronous airgap harmonics, making them susceptible to rotor eddy-current losses. These losses can be reduced by using novel high resistivity materials like plastic bonded magnets and carbon fiber reinforced retaining sleeves. This paper examines by numerical studies the consequences for the losses of using these materials. The effect of laminating the magnets and the losses in the anisotropic sleeve are analysed with 3D simulations, using simplifications determined with 2D simulations. The anisotropic resistivity of the sleeve roughly doubles the losses compared to an isotropic sleeve, while laminating the magnets in $n$ slices reduces the losses by $1 / n^{1.8}$.
\end{abstract}

\section{Introduction}

High speed permanent magnet machines are increasingly popular because they can directly drive high speed loads and have a high power density [5]. With proper modifications they can be made fault-tolerant, allowing the use in safetycritical applications such as airplanes as well [4]. Due to the high rotational speeds both air friction and electrical losses increase and because these machine are rather small, the loss density is potentially higher than in conventional speed machines. The rotor eddy-current losses are a particular point of concern because only air or liquids passing through the airgap can be used for cooling. In fault-tolerant machines these losses are even more severe, due to strong asynchronous space-harmonics.

In [8] a $50 \mathrm{~kW}, 60,000 \mathrm{RPM}$, high speed fault tolerant machine using air to cool the rotor is presented. With conventional materials like sintered magnets and a metallic retaining sleeve the rotor losses in a similar machine would be in the order of several kilowatts [1]. The authors in [1] intend to submerge the entire machine in aviation fuel, which has excellent cooling properties, but has implementation difficulties. Air is much less effective however and a safe rotor temperature cannot be maintained at this loss level. To reduce the losses to a level that can be cooled with air, a carbon fiber retaining sleeve and laminated plastic bonded magnets are used. In analytical 2D computations the high resistivity of these materials reduces the rotor losses to an acceptable level.
Replacing a metal retaining sleeve with a carbon fiber reinforced plastic (CFRP) type is not a new concept [5] and loss reduction has been reported in [2,12]. When analyzing the losses most authors use the resistivity of the carbon fibers, which is approximately $20-40 \mu \Omega \mathrm{m}$, in their computations. Even though this produces a pessimistic view on the effectiveness, a substantial reduction is observed.

An actual retaining sleeve may have a strong anisotropic resistivity however, due to the mechanical construction. Since the mechanical forces in the sleeve are predominantly in the radial direction the majority of the fibers are placed in the hoop direction. The carbon fibers are much better conductors than the binding resin and a strong anisotropic resistivity results. In [7] this anisotropy is taken into account in an analytical computation with 2D magnetic fields, but this is not applied to an actual machine.

To further reduce the rotor losses the usual sintered magnets, with a resistivity in the order of $1 \mu \Omega \mathrm{m}$, have been replaced with plastic bonded magnets which have a resistivity of about $10-200 \mu \Omega \mathrm{m}$, but only half the remanent flux density. Without physical modifications the rotational speed would have to be doubled to restore the power density, potentially resulting in four times the rotor losses, but the much higher resistivity still yields a net reduction of the losses. Segmenting the magnets, both in hoop and axial direction, is another effective means to reduce the losses [11]. The magnets in this machine are fabricated as complete rings, and only axial segmentation (lamination) is used.

In this paper the effect on the rotor losses of using an anisotropic retaining sleeve and laminated plastic bonded magnets will be investigated with numerical 3D simulations. The effect of magnet segmentation is also analysed with 3D analyses in [3,6], but they do not consider an (anisotropic) sleeve and use $2 \mathrm{D}$ boundary conditions. In this work $3 \mathrm{D}$ boundary conditions are used. $2 \mathrm{D}$ simulations are used first to determine which effects have to be included in the 3D simulations, after which only the rotor is modelled with $3 \mathrm{D}$ time-harmonic simulations. This approach is very similar to [6], although they include an effective airgap to correctly account for the skin-effect. This may be omitted if the eddycurrents are resistance limited 


\section{Considered Machine}

To investigate the losses a conceptual $50 \mathrm{~kW}, 60 \mathrm{kRPM}$ fault tolerant machine is used [8]. All relevant specifications are listed in Table 1.

\subsection{The stator}

Figure 1 shows a cross section of the stator. Each of the six phases is wound around a single tooth. A separation tooth between the phases provides electrical, thermal and magnetic isolation, a requirement for fault tolerance [4]. The concentrated windings produce a very rich airgap field containing space harmonics of order $4 \pm 6 n, n=0,1,2,3, \ldots$, where negative values rotate opposite to positive values. The fourth space harmonic (with eight poles) is synchronous with the rotor.

Each phase is designed with a 1 per unit inductance -another requirement for a fault tolerant PM machine- acting as a filter to time-harmonics in the inverter output voltage. This may allow certain time-harmonics in the phase current to be ignored in analyses.

\begin{tabular}{|l|l|}
\hline Property & Value \\
\hline Power & $50 \mathrm{~kW}$ \\
\hline Speed & $60,000 \mathrm{RPM}$ \\
\hline Phases & 6 \\
\hline Poles & 8 \\
\hline
\end{tabular}

Table 1: Basic specifications of the machine considered

\subsection{The Composite Materials Rotor}

In Figure 2 the composite rotor is shown. A CFRP retaining sleeve is interference fitted on a stack of plastic bonded magnet rings which are mounted on a steel shaft. The resistivity of the sleeve is determined to be $20 \mu \Omega \mathrm{m}$ along the fibers $\left(\rho_{t}\right)$ and at least $1 \mathrm{~m} \Omega \mathrm{m}$ perpendicular to the fibers $\left(\rho_{z}\right)$, in the direction of the machine axis [9]. The resistivity in radial direction is assumed equal to $\rho_{\mathrm{t}}$. In reality it will be somewhere between the tangential and axial resistivity, but the selected value gives the most pessimistic case.

The axial resistivity, $\rho_{\mathrm{z}}$, is expected to be most important to the rotor losses because in a radial flux machine the majority of the eddy-currents are induced in this direction. Compared to metals this resistivity is three to four orders of magnitude larger, which should substantially reduce the losses. Downsides of CFRP are that the resin weakens when it heats up, limiting the maximum operating temperature, and a poor thermal conductivity, potentially causing higher magnet temperatures than with a metallic retaining sleeve. Furthermore, a CFRP sleeve does not shield the magnets from any space harmonics, possibly leading to more losses deeper inside the rotor.

The plastic bonded magnets are fabricated by mixing conductive magnetic particles with an insulating filler material. By carefully choosing the ratio of the magnet and filler material, a balance may be obtained between a high

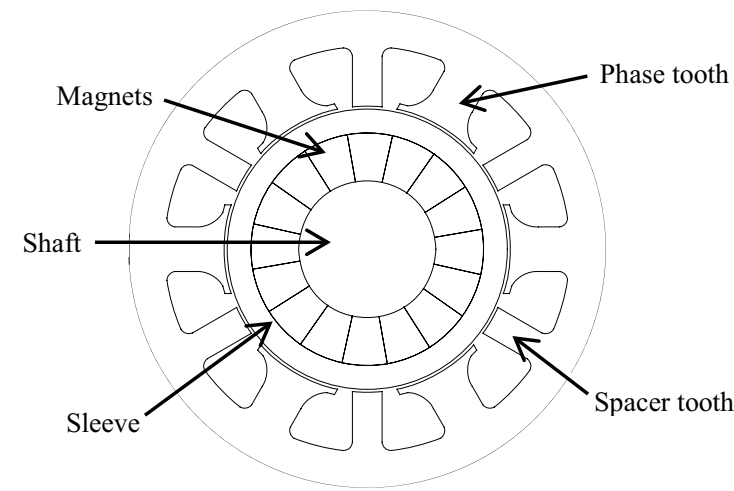

Figure 1: Cross-section of the conceptual machine

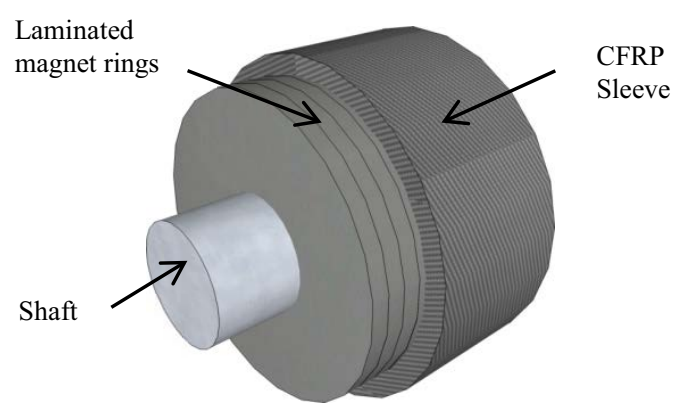

Figure 2: Impression of the conceptual rotor

remanent flux density on the one hand and a high resistivity on the other hand. The magnets considered here have a measured resistivity of approximately $200 \mu \Omega \mathrm{m}$.

To further reduce the losses the magnets may be segmented as well. Since eddy-currents are induced in axial direction in a radial field machine, segmentation in axial direction (i.e. lamination) should be the most effective orientation. However, tangentially segmenting the magnets may also reduce the losses [11], especially if the segments are shorter than the tangential length of the main loss inducing space harmonics.

For this machine the magnets are fabricated as complete rings, making axial segmentation more practical. If barmagnets were used tangential segmentation may also be an attractive solution.

\section{2D Analysis}

Both the magnet lamination and sleeve anisotropy cannot directly be taken into account with $2 \mathrm{D}$ simulations. 2D simulations execute much faster however, and are therefore used to determine which effects have to be included in the 3D simulations. No analytical or empirical corrections for the finite length or laminations are therefore utilized.

The computing costs can always be decreased somewhat by exploiting symmetries in the machine, but with timeharmonic simulations of only the rotor instead of transient simulations with a full model, a much larger speed up can be obtained. This requires all interactions with the stator to either be included as boundary conditions, or be of negligible magnitude. 


\begin{tabular}{|l|l|}
\hline \multicolumn{2}{|c|}{ Retaining sleeve } \\
\hline Resistivity & $\begin{array}{l}\text { Hoop direction }\left(\rho_{\mathrm{t}}\right): 20 \mu \Omega \mathrm{m} \\
\text { Axial direction }\left(\rho_{\mathrm{z}}\right):>1 \mathrm{~m} \Omega \mathrm{m}\end{array}$ \\
\hline Relative permeability & 1 \\
\hline \multicolumn{2}{|c|}{ Plastic bonded magnets } \\
\hline Resistivity & $200 \mu \Omega \mathrm{m}$ \\
\hline Relative permeability & 1.2 \\
\hline
\end{tabular}

Table 2: Electrical properties of the rotor materials

Transient 2D simulations will now be used to determine which of the following interactions have to be included in time-harmonic 3D simulations:

--Space harmonics

--Stator slotting

--Skin effect

--Magnet relative recoil permeability

--Stator current time-harmonics

\section{Space harmonics}

For each space-harmonic a separate time-harmonic simulation is required, so only harmonics inducing significant rotor losses should be considered. To determine which spaceharmonics are relevant the eddy-current losses due to each harmonic are computed. The space-harmonic spectrum of the radial magnetic field along the airgap, computed with the Fourier transform, is shown in Figure 3. Since timeharmonics were neglected the amplitudes are static in time. Many harmonics are clearly present, but the $2^{\text {nd }}$ and $4^{\text {th }}$ are particularly strong. However, most higher order harmonics decay rapidly with decreasing radius and only the $2^{\text {nd }}$ spaceharmonic is present throughout the entire rotor.

The relative power loss due to each harmonic, computed by squaring the harmonic amplitudes of the currents induced in the rotor, is shown in Figure 4 on the outer edge of the rotor. Despite the rich airgap field most losses are due to the $2^{\text {nd }}$ harmonic. By performing this computation at several radii in the rotor the relative power loss due to each harmonic can be computed. Figure 5 shows the result; in the magnets $99.6 \%$ and in the sleeve $86.8 \%$ of the total power loss is caused by the $2^{\text {nd }}$ space harmonic. In the $3 \mathrm{D}$ simulation only this harmonic will therefore be considered.

\section{Stator slotting}

Stator slotting is a variation in the working point of the magnets due to the changing magnetic path as the rotor rotates. Due the 1-per-unit inductance requirement this machine has rather small tooth openings, which limits the effect of stator slotting. At rated speed and without stator currents the rotor losses are $0.74 \mathrm{~W}$, which allows stator slotting to be ignored.

\section{Skin effect}

If eddy-currents are sufficiently large they interact with the inducing field, thereby reducing the field strength inside and near the rotor. This is roughly the case when the skin-depth becomes smaller than the geometry. In models with only the rotor an equivalent airgap has to be used to correctly account for the skin-effect, if present [6]. The skin-depth for the lower

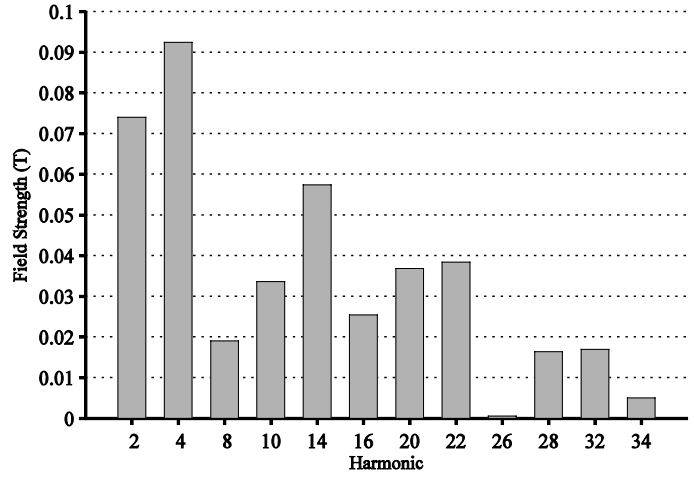

Figure 3: Airgap space harmonic spectrum. The fourth harmonic is synchronous with the rotor

order space-harmonics is given in Table 3; all of them are larger than the magnet or sleeve thickness and the eddycurrents are resistance limited. Moreover, lamination increases the effective skin-depth. Hence, the eddy currents are entirely resistance limited.

\section{Magnet permeability}

In many analyses the magnet relative permeability is taken to be unity. The magnets used have a relatively high relative permeability however, and some simulations were performed to investigate the consequences. In Fig. 6 the losses as function of $\mu_{\mathrm{r}}$ computed with a transient 2D simulation are shown. The computed values are not scaled for any end-effect and do contain useful information, but the trend should be independent of the absolute value. A strong, almost linear dependency is observed for both the magnet and sleeve losses. This is explained by more flux being attracted to the rotor and passing the airgap. At $\mu \mathrm{r}=1.2$ the losses are some $20 \%$ higher, and the relative permeability should certainly be included in the $3 \mathrm{D}$ simulations.

\section{Time-harmonics}

The machine will be fed by a square wave supply voltage with only the 1 per unit phase inductance acting as a filter [10]. Therefore the phase currents will not be perfectly sinusoidal; an example of the expected phase current in shown in Figure 6. To determine which time-harmonics have to be included the total rotor losses due to all relevant timeharmonics are computed.

For every time-harmonic a separate transient simulation with only that harmonic applied is used. The result is shown in Table 4. It is immediately clear that non-fundamental timeharmonics may be ignored, since some $96 \%$ of the rotor losses are by the fundamental phase current.

\begin{tabular}{ccc}
\hline $\begin{array}{c}\text { Space } \\
\text { harmonic }\end{array}$ & $\begin{array}{c}\text { Magnet skin } \\
\text { depth }(\mathbf{m m})\end{array}$ & $\begin{array}{c}\text { Sleeve skin } \\
\text { depth }(\mathbf{m m})\end{array}$ \\
\hline 2 & 84 & 27 \\
4 & (Synchronous) & (Synchronous) \\
8 & 59 & 19 \\
10 & 84 & 27 \\
14 & 48 & 15 \\
16 & 59 & 19 \\
20 & 42 & 13 \\
22 & 48 & 15 \\
\hline
\end{tabular}

Table 3: Skin depth for various space harmonics 


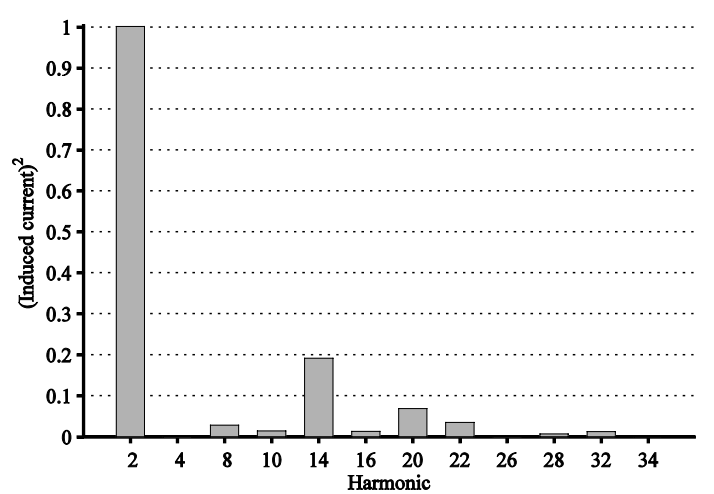

Figure 4: Relative power loss due to each harmonic at the rotor surface. A few $\mathrm{mms}$ into the rotor only the $2^{\text {nd }}$ harmonic remains

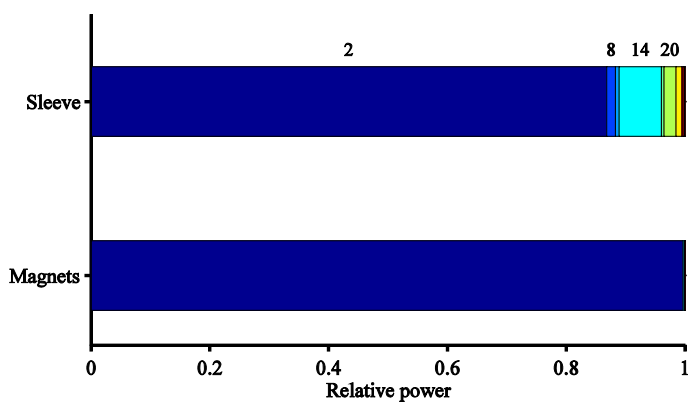

Figure 5: Total rotor losses due to each space harmonic

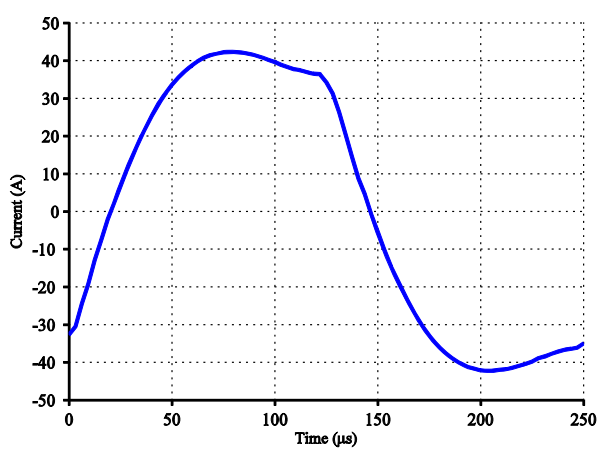

Figure 6: Expected phase current waveform.

\begin{tabular}{|c|c|}
\hline Time harmonic & Fraction of rotor losses \\
\hline 1 & $96.3 \%$ \\
\hline 3 & $0.4 \%$ \\
\hline 5 & $1.9 \%$ \\
\hline 7 & $1.1 \%$ \\
\hline 9 & $0.1 \%$ \\
\hline 11 & $0.2 \%$ \\
\hline
\end{tabular}

Table 4: Rotor losses caused by individual time-harmonics

\section{3D Analysis}

The simplifications obtained from 2D simulations show it is possible to obtain reasonably accurate loss figures using a time-harmonic simulation with only the rotor geometry and the second space harmonic. This forms the basis for the $3 \mathrm{D}$ simulations executed in this section.

\subsection{Simulation approach}

By using 3D simulations the losses in the anisotropic sleeve

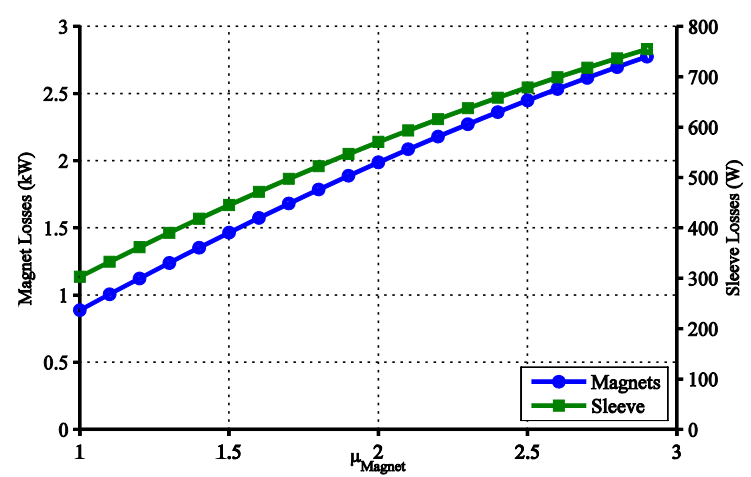

Figure 7: Non-scaled magnet and sleeve losses as a function of $\mu_{\mathrm{r}, \text { magnet }}$

and laminated magnets can be accurately computed. Transient 3D simulations can take a long time to solve however, and a two-step approach is employed to minimize computation time:

1. A static simulation of the complete machine is solved with the equation:

$$
\nabla \times \frac{1}{\mu} \times \boldsymbol{A}=J_{\boldsymbol{e}}
$$

where $\boldsymbol{A}$ is the magnetic vector potential and $\boldsymbol{J}_{\boldsymbol{e}}$ represents the phase currents. The individual components of $\boldsymbol{A}$ at the rotor periphery are then decomposed into space-harmonics, after which the dependency on $r$ and $z$ of the $2^{\text {nd }}$ harmonic is determined.

2. Time harmonic simulations containing only (parts of) the rotor are solved with the boundary conditions computed in step 1. The equations solved are (omitting negligible components):

$$
\begin{aligned}
\nabla \times \frac{1}{\mu} \times \boldsymbol{A}+\sigma(j \omega \boldsymbol{A}-\nabla \phi) & =0 \\
\nabla \cdot \sigma(j \omega \boldsymbol{A}+\nabla \phi) & =0
\end{aligned}
$$

where $\phi$ is the scalar electrical potential. This approach is justified by the fact that the eddy-currents are resistance limited and the losses due to stator slotting are negligible. Moreover, since only one space harmonic is responsible for most of the losses, only one time-harmonic simulation is required per geometry.

\subsection{Effect of magnet lamination}

Lamination can very effectively reduce eddy-current losses; theoretically the losses decrease by the square of the number of lamination slices. In reality a part of the field may be perpendicular to the slice and decrease the effectiveness. To examine the effect of lamination the solid magnets are divided in $n$ slices and each of them is simulated separately. Separate boundary condition are used for every slice, depending on the position of the slice in the rotor. In this way the axial dependency of the field strengths towards the ends of the rotor is also accounted for.

Although this approach inherently assumes a perfect insulation between the slices, it does allow very thin slices to 
be modelled, which would otherwise require too much memory. An infinitesimally thin insulation was assumed, although any thickness could be implemented. Figure 8 shows a magnet slice with part of the mesh and flow patterns of the eddy currents. At least 4 elements in the z-direction were used.

The losses were computed for a rotor with 1 to 36 magnet slices and the results are shown in Figure 9. Without lamination the magnet losses are about $600 \mathrm{~W}$. With 6 or more slices, laminating the magnets reduces the losses by $1 / n^{1.8}$ in this machine, making it an effective loss reduction method. Although Fig. 8 suggests that the losses may be reduced well below $10 \mathrm{~W}$, they may be higher in reality due to non-perfect insulation and leakage currents at the sides of the magnets.

\subsection{Effect of sleeve Anisotropy}

The used sleeve exhibits a strong anisotropy, and the effect on the losses will now be computed. The resistivity in the axial direction is held constant, while the resistivity in radial and tangential directions is equal and varied. This is repeated for several values of $\rho_{z}$ and the results are shown in Figure 10.

At $\rho_{\mathrm{z}}=1 \mathrm{~m} \Omega \mathrm{m}$, the lowest anticipated value, the losses would be $125 \mathrm{~W}$ for an isotropic sleeve and $240 \mathrm{~W}$ when $\rho_{\mathrm{r}}$ $=20 \mu \Omega \mathrm{m}$. This clearly shows that the anisotropic cannot be ignored.

In general, for realistic values of $\rho_{\mathrm{r}}$ and $\rho_{\mathrm{t}}(10-100 \mu \Omega \mathrm{m})$ the dependency of the losses on $\rho_{z}$ is much larger than on $\rho_{\mathrm{r}}$ and $\rho_{\mathrm{t}}$ : An eightfold loss reduction occurs with a tenfold increase in $\rho_{\mathrm{z}}$, while a tenfold increase in $\rho_{\mathrm{r}, \mathrm{t}}$ causes no significant loss reduction. In this light it is somewhat unfortunate that the value of $\rho_{t}$ is known more accurately than $\rho_{z}$.

\section{Conclusion}

In this paper the losses in the magnets and retaining sleeve of the rotor of a high speed fault tolerant machine were computed by means of $3 \mathrm{D}$ simulations. Time-harmonic simulations were used, using boundary conditions obtained from a static simulation.

A CFRP sleeve with anisotropic resistivity was considered. The anisotropy increases the losses compared a theoretical high resistivity sleeve, but the resistivity in axial direction is governing for the losses. The loss reduction due to laminating

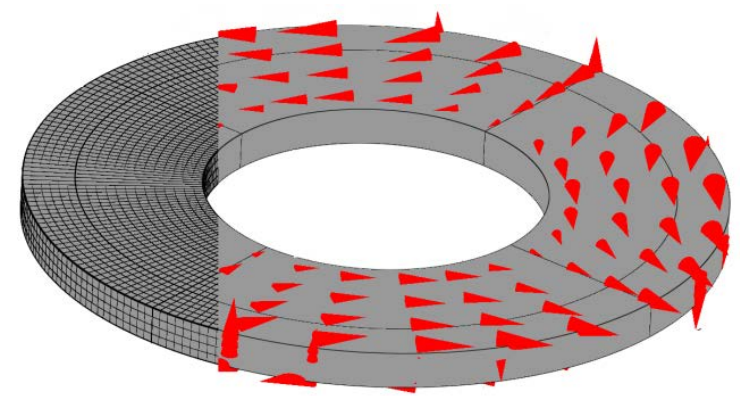

Figure 8: A magnet slice with the mesh (left) en eddy-current pattern. Note the four pole nature of the eddy-currents high resistivity magnets was also considered. In solid magnets the magnet losses would be $600 \mathrm{~W}$. With lamination a reduction of $1 / n^{1.8}$ was observed, $n$ being the number of slices, which allows the losses to be reduced to less than $10 \mathrm{~W}$ in an ideal case.

The next step is the measurement of the actual losses to validate the simulations. The machine described here is currently under construction and will undergo testing when ready.

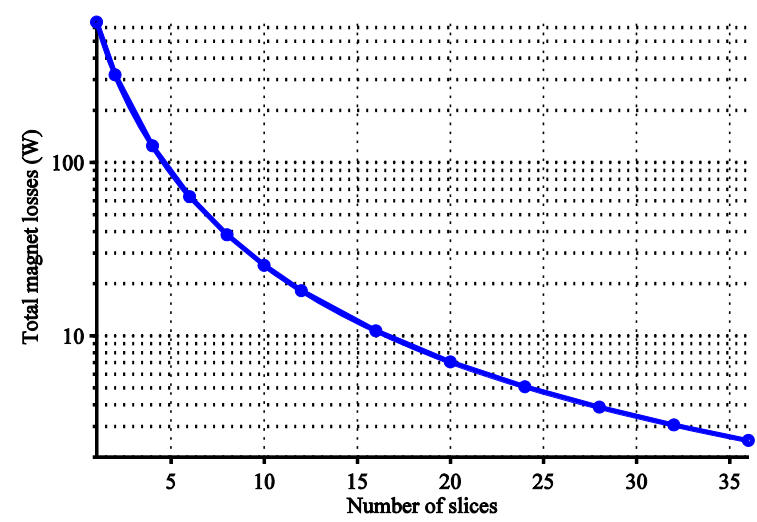

Figure 9: Magnet loss versus the number of slices. 1 'slice' is the power for solid magnets

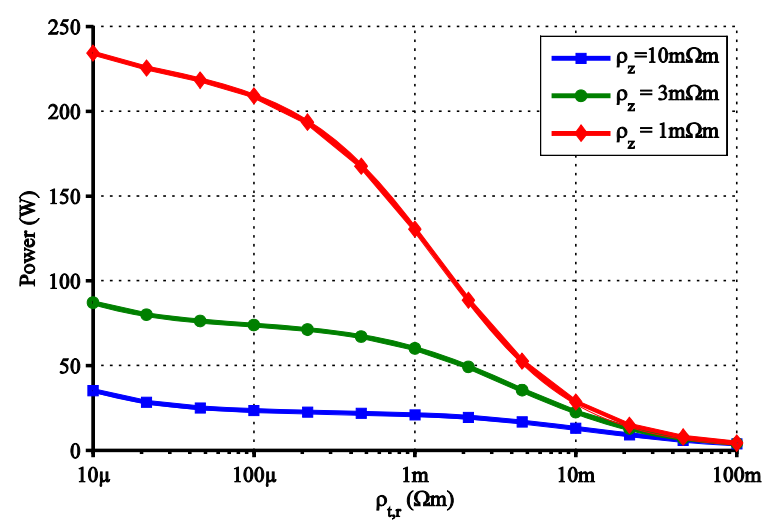

Figure 10: The losses in the sleeve as a function of radial and tangential resistivity for three different axial resistivities

\section{References}

[1] G.J. Atkinson, B.C. Mecrow, A.G. Jack, D.J. Atkinson, P. Sangha, M. Benarous, "The Analysis of Losses in High-Power Fault-Tolerant Machines for Aerospace Applications", IEEE Trans. Ind. Appl., vol.42, no.5, pp.1162-1170, (2006).

[2] Han-Wook Cho, Seok-Myeong Jang, Sang-Kyu Choi, "A Design Approach to Reduce Rotor Losses in HighSpeed Permanent Magnet Machine for TurboCompressor," IEEE Trans. Magn., vol.42, no.10, pp.3521-3523, (2006).

[3] J.D. Ede, K. Atallah, G.W. Jewell, J.B. Wang, D. Howe, "Effect of Axial Segmentation of Permanent Magnets on Rotor Loss in Modular Permanent-Magnet Brushless 
Machines", IEEE Trans. Ind. Appl., vol.43, no.5, pp.1207-1213, (2007).

[4] A.G. Jack, B.C. Mecrow, J.A. Haylock, "A comparative study of permanent magnet and switched reluctance motors for high-performance fault-tolerant applications", IEEE Trans. Ind. Appl., vol.32, no.4, pp.889-895, (1996).

[5] Z. Kolondzovski, A. Arkkio, J. Larjola, P. Sallinen, "Power Limits of High-Speed Permanent-Magnet Electrical Machines for Compressor Applications", IEEE Trans. Energy Convers., vol.26, no.1, pp.73-82, (2011).

[6] T. Okitsu, D. Matsuhashi, K. Muramatsu, "Method for Evaluating the Eddy Current Loss of a Permanent Magnet in a PM Motor Driven by an Inverter Power Supply Using Coupled 2-D and 3-D Finite Element Analyses", IEEE Trans. Magn., vol.45, no.10, pp.45744577, (2009)

[7] B. Pratap, W.F. Weldon, "Eddy currents in anisotropic composites applied to pulsed machinery", IEEE Trans. Magn., vol.32, no.2, pp.437-444, (1996).
[8] J. Wolmarans, H. Polinder, J.A. Ferreira, D. Clarenbach, "Design of a fault tolerant permanent magnet machine for airplanes", Int. Conf. Electrical Machines and Systems, pp.2882-2887, (2008)

[9] J.J Wolmarans, M. van der Geest, H. Polinder, J.A. Ferreira, D. Zeilstra, "Composite Materials for Low Loss Rotor Construction”, Int. Conf. Elec. Mach. Driv. (2011).

[10] J.J. Wolmarans, H. Polinder, J.A. Ferreira, D. Zeilstra, "A fault tolerant drive for high speed permanent magnet machines", EPE 2011, (2011).

[11] K. Yamazaki, Y. Fukushima, "Effect of Eddy-Current Loss Reduction by Magnet Segmentation in Synchronous Motors With Concentrated Windings", IEEE Trans. Ind. Appl., vol.47, no.2, pp.779-788, (2011).

[12] Fengzheng Zhou, Jianxin Shen, Weizhong Fei, Ruiguang Lin, "Study of Retaining Sleeve and Conductive Shield and Their Influence on Rotor Loss in High-Speed PM BLDC Motors", IEEE Trans. Magn., vol.42, no.10, pp.3398-3400, (2006). 